\title{
A Study on Using Reclaimed Asphalt Pavement (RAP) in the Construction of New Roads in the State of Qatar
}

\author{
K. Lakshmi Roja \\ lakshmi.kakumanu@qatar.tamu.edu \\ Mechanical Engineering Program, Texas A\&M University at Qatar, Doha, Qatar \\ Eyad Masad \\ eyad.masad@qatar.tamu.edu \\ Mechanical Engineering Program, Texas A\&M University at Qatar, Doha, Qatar
}

\begin{abstract}
In the State of Qatar, road engineers started using RAP in the base course of asphalt pavement from past few years. The use of RAP not only replaces the quantity of virgin aggregate and binder, it also improves the overall stiffness of asphalt mixture due to the addition of aged asphalt binder. Considering the economic and environmental benefits of RAP, in this study, a mix design was developed for asphalt mixtures containing different proportions of RAP and evaluate the performance of RAP blended asphalt mixtures. Based on obtained properties, it was attempted to optimize the use of RAP content in the base course of asphalt pavement. In this study, the asphalt mixtures were produced with three different RAP contents $(15,25$ and 35\%) and the behavior of these mixtures was benchmarked with conventional mix ( $0 \%$ RAP). The RAP blended asphalt mixtures were tested using several experimental protocols. First, the stiffness modulus of RAP mixtures were determined using AASHTO test method. The high and low temperature distress characteristics of mixtures were determined using Hamburg wheel tracker and semi-circular beam bending test. Based on the stiffness and performance properties, a suitable RAP content and the ways to increase the use of RAP content was recommended.
\end{abstract}

Keywords: Reclaimed asphalt pavement; Mix design; Rutting; Fracture; Optimum RAP content

\section{INTRODUCTION}

Large scale road projects are being constructed in the State of Qatar for the past few years. The construction of new road projects requires large quantities of aggregate and binder. Using RAP in the asphalt mix design can be an alternate solution to reduce the consumption of natural resources. Nowadays, the usage of RAP in pavement construction is tremendously increasing due to the benefits of this material in terms of improved performance, cost effectiveness and reduced environmental impact. However, the high and low temperature performance characteristics of RAP blended asphalt mixture are highly dependent on the properties of RAP binder and aggregate. The two major distresses in asphalt pavements are rutting and fatigue cracking. The addition of RAP to virgin asphalt mixture may increase the total stiffness of mix, which may cause higher resistance to rutting and poor resistance to fatigue cracking (Al-Qadi et al., 2007; Shu et al., 2008). While using RAP in the asphalt mix design, it is necessary to balance both high and low temperature distress properties. 
To allow high RAP contents in asphalt mix design, few researchers used rejuvenators and adjusted the mix design slightly to improve the resistance to fracture (Mogawer et al., 2015; Menapace et al., 2018). The optimum RAP content can be determined by balancing the performance of asphalt mixtures containing different proportions of RAP at critical pavement service temperatures. The objective of this study was to evaluate the effect of using highly oxidized asphalt on the mixture performance. This objective was achieved by conducting the following tasks:

- Develop a mix design for RAP blended asphalt mixtures and maintain the similar volumterics for virgin and RAP blended asphalt mixtures.

- Evaluate the high and low temperature distresses of asphalt mixtures containing RAP.

- Recommend to the State of Qatar an allowable RAP content that will not impact the durability of asphalt mixtures negatively.

\section{MATERIALS AND MIX DESIGN}

\subsection{RAP characterization}

A local contractor in Qatar supplied the RAP material. For asphalt mix design, the RAP material passing $12.5 \mathrm{~mm}$ sieve was considered. The RAP binder was extracted and recovered using rotary evaporator as per ASTM D 7906 (2014). The true (continuous) grade of the recovered binder was PG 94-0. These PG high and low temperatures were exact pass/fail temperatures obtained from $\mathrm{G}^{*} / \sin \delta$ values and Bending Beam Rheometer (BBR) testing. The basic properties of the RAP recovered binder and aggregates were determined. From the literature (Sreeram et al., 2018), it was assumed that only $70 \%$ of RAP binder was effective for the production of asphalt mixtures.

\subsection{Production of asphalt mixtures}

A base course class A gradation (Qatar Construction Specification, 2014) with a nominal maximum aggregate size (NMAS) of $25 \mathrm{~mm}$ was selected to produce these mixtures. Four types of asphalt mixtures were produced: control mix ( $0 \%$ RAP), 15, 25 and $35 \%$ RAP blended mixtures. The superpave mix design was used for all mixtures. A base binder of PG 64-22 (Pen 60/70) grade was selected in this study. For the production of asphalt mixtures, five aggregate stockpiles $37.5-22 \mathrm{~mm}, 22-12 \mathrm{~mm}, 12-8 \mathrm{~mm}, 8-4 \mathrm{~mm}$, $<4 \mathrm{~mm}$ and RAP stockpile of $<12.5 \mathrm{~mm}$ and filler material were used.

The virgin aggregates and RAP material were heated separately at $160^{\circ} \mathrm{C}$ and $110^{\circ} \mathrm{C}$ respectively and they were mixed with binder at a temperature of $153^{\circ} \mathrm{C}$. For testing mechanical properties, asphalt mixtures were short-term aged for 4 hours at $135^{\circ} \mathrm{C}$ and then increased to a compaction temperature (AASHTO R30, 2011). These samples were compacted using gyratory compactor to a traffic level of $>30$ million ESAL $\left(\mathrm{N}_{\text {design }}=125\right.$ gyrations). The size of gyratory samples was $150 \mathrm{~mm}$ diameter and $165 \mathrm{~mm}$ height and the target air voids of $7 \pm 0.5 \%$ were used for all tests in this study.

\subsection{Mix design}

All the mixtures were prepared with binder contents of 2.5, 3.0, 3.5, 4.0 and 4.5\%. Based on the values of stability, flow, Voids in Mineral Aggregates (VMA), Voids Filled with Asphalt (VFA) and air void, the optimum binder contents of 0, 15, 25 and 35\% RAP 
blended mixtures were determined as 3.9, 3.7, 3.5 and 3.5\%, respectively. For all the mixtures, the VMA values were maintained in between $12-12.5 \%$ and the VFA values were in between $67-70 \%$ (as per QCS, 2014).

\section{MATERIAL PROPERTY- DYNAMIC MODULUS}

The dynamic modulus tests were conducted using an Asphalt Mixture Performance Tester (AMPT) in confined condition (AASHTO T378-17, 2017). The sinusoidal loading waveform was applied with confinement pressure of $69 \mathrm{kPa}$ and the measured strains were in between 85 to 115 microstrain. The tests were conducted at five different temperatures of $5,15,25,35$ and $45^{\circ} \mathrm{C}$ and three frequencies of $0.1,1$ and $10 \mathrm{~Hz}$. The dynamic modulus $\left(\left|\mathrm{E}^{*}\right|\right)$ and phase lag $(\delta)$ values were calculated from the ratio of peak stress to peak strain and the time lag between stress and strain curves (AASHTO T378-17, 2017). From the obtained $\left|E^{*}\right|$ values, the master curves were constructed at a reference temperature of $35^{\circ} \mathrm{C}$ as shown in Figure 1. It was observed that the addition of RAP increased the stiffness of asphalt mixtures. However, it is necessary to analyze the performance characteristics of such mixtures to identify an optimum RAP content for asphalt mix design.

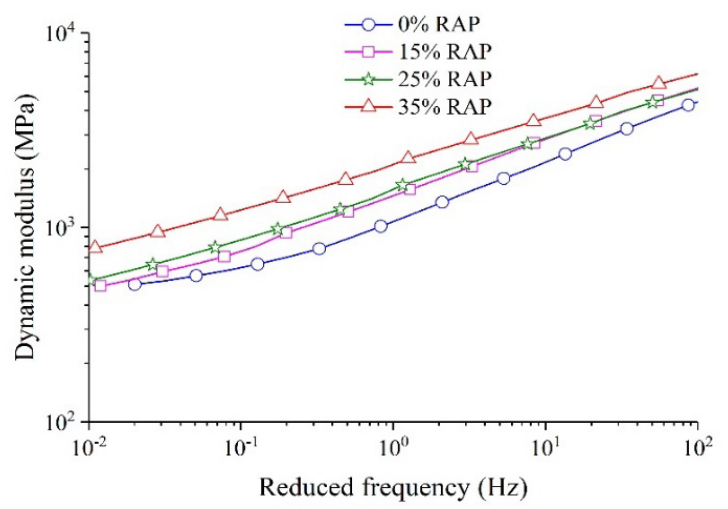

Figure 1: Master curves for RAP blended asphalt mixes

\section{RUTTING CHARACTERIZATION}

In this study, two test protocols were used to characterize the rutting: Hamburg Wheel Tracker (HWT) and Flow Number (FN) test. In HWT test, a wheel load of $700 \mathrm{~N}$ was passed on the surface of the sample in to and from direction at a speed of 52 passes per minute (AASHTO T 324-14, 2014). The rut depth was measured along wheel path using a Linear Variable Differential Transducer (LVDT) and the test was terminated at 20000 passes. For each mix, total of four samples were mounted in the testing device: two on left side, two on right side. The test was conducted in dry condition at $64^{\circ} \mathrm{C}$ and wet condition at $50^{\circ} \mathrm{C}$. The average of maximum rut depth on both left and right sides are plotted in Figure 2 for both dry and wet conditions. The addition of RAP content caused an increase in rut resistance. In dry condition, the final rut depth for 0, 15, 25 and 35\% RAP blended mixes was measured as 17.1, 14.1, 8.6, and $6.2 \mathrm{~mm}$, respectively (Figure 2(a)) whereas, in wet condition, the behavior of all the RAP blended asphalt mixes (15, 25 and $35 \%$ ) was more or less same and the final rut depth was less than $12.5 \mathrm{~mm}$ for all the mixes (Figure 2(b)). 


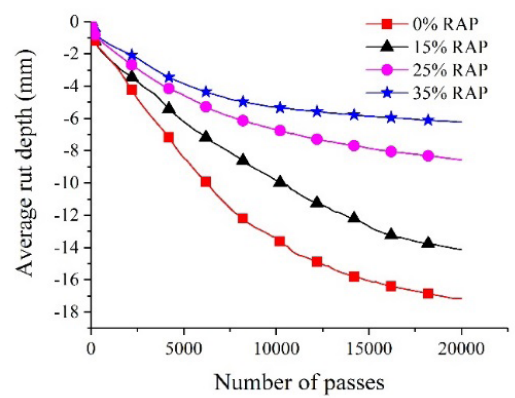

(a) Dry condition

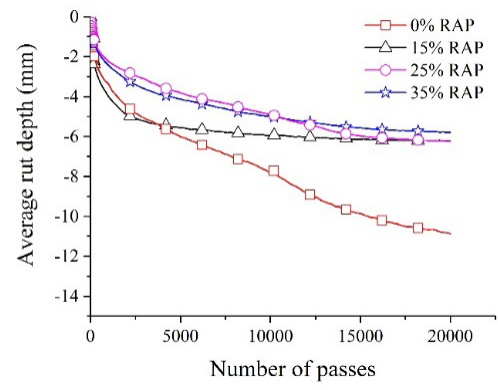

(b) Wet condition

Figure 2: Rut depth of asphalt mixtures containing different proportions of RAP

A standard flow number test method was carried out using an Asphalt Mixture Performance Tester (AMPT). A deviatoric stress of $600 \mathrm{kPa}$ was applied with a confinement level of $69 \mathrm{kPa}$ at a temperature of $40^{\circ} \mathrm{C}$. The test consists of $0.1 \mathrm{~s}$ haversine load and $0.9 \mathrm{~s}$ rest period (AASHTO T378-17, 2017). The test was terminated at 10000 cycles. The accumulated irrecoverable deformation at the end of each cycle was recorded and shown in Figure 3. Out of all the mixes, virgin sample ( $0 \%$ RAP) showed more susceptibility to rutting and it has higher percentage of strain. The mix with higher RAP content $(35 \%)$ has greater rut resistance and the similar observation was seen from wheel tracking tests on mixtures. Flow number is defined as the number of cycles corresponding to the starting of tertiary stage and it can be identified from the rate of change of slope of irrecoverable strain using Francken model (Equation 1) (Francken, 1977).

$$
\varepsilon_{p}=a N^{b}+c\left(\exp ^{d N}-1\right)
$$

where, $\varepsilon_{\mathrm{p}}$ represents the irrecoverable strain, $\mathrm{N}$ represents the number of load cycles and $\mathrm{a}, \mathrm{b}, \mathrm{c}$ and $\mathrm{d}$ represents model parameters. The parameters a and b capture the primary and secondary stage and $\mathrm{c}$ and d capture the tertiary stage. In Figure 3, it can be observed that there is no sample failure within the test duration ( $c=0$ for no tertiary stage). The first term of equation 1 can be used to capture the primary and secondary stages of (parabolic shape) of three stage creep curve. Parameters a and b derived from the linear portion of primary and secondary stage of the accumulated strain are shown in Figure 3. For all the mixes, irrecoverable strain accumulated at almost same rate. The parameters obtained for 15 and $25 \%$ RAP mixes were identical.

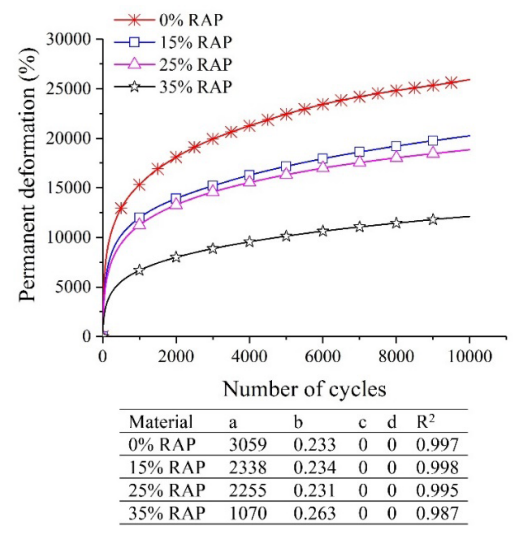

Figure 3: Creep curves for asphalt mixes containing different proportions of RAP and Francken model parameters 


\section{FRACTURE RESISTANCE}

Fracture resistance of asphalt mixtures is an indicator of fatigue behavior that occurs due to the accumulation of tensile strains during repeated loading. In this study, the fracture resistance of RAP contained mixtures was evaluated using Semi Circular Bend (SCB) test. SCB tests were conducted on gyratory samples of thickness $50 \pm 2 \mathrm{~mm}$ and these circular samples were cut into two equal halves. The selected notch depth and width were $16 \mathrm{~mm}$ and $3 \mathrm{~mm}$, respectively. A vertical load was applied on these samples with a constant displacement rate of $0.5 \mathrm{~mm} / \mathrm{min}$ (Sreedhar et al., 2018). When the applied load was below $0.5 \mathrm{kN}$, the test was terminated. The selected test temperature was $25^{\circ} \mathrm{C}$. At least four specimens were tested for each type of mix to check the repeatability.

In the load-displacement curve (Figure 4), the area under curve was calculated using equation 2 which represents the 'work of fracture'. From which, the fracture energy and Flexibility Index (FI) were calculated using equation 4 and 5, respectively. FI parameter is mainly used for fatigue performance evaluation and it is defined as the ratio of the fracture energy $\left(G_{f}\right)$ to the slope at post-peak inflection point of load-displacement curve. FI parameter can dictate the brittleness of material and the lower value of the material shows more brittleness with a higher crack growth rate (Ozer, 2016).

$$
\begin{array}{lc}
\text { Work of fracture } W_{f}=\int P d u & \text { (in } \mathrm{kJ}) \\
\text { Ligment area } A=(r-a) \times t & \left(\text { in } \mathrm{m}^{2}\right) \\
\text { Fracture energy } G_{f}=\frac{W_{f}}{A} & \text { (in } \left.\mathrm{kJ} / \mathrm{m}^{2}\right) \\
\text { Flexibility index } F I=\frac{G_{f}}{a b s(m)} \times 0.01 &
\end{array}
$$

where $\mathrm{P}=$ applied load $(\mathrm{kN}), \mathrm{u}=$ load line displacement $(\mathrm{m}), \mathrm{r}=$ sample radius $(\mathrm{m})$, $\mathrm{a}=$ notch length $(\mathrm{m})$, and $\mathrm{t}=$ sample thickness $(\mathrm{m}), \mathrm{abs}(\mathrm{m})=$ absolute value of the slope at post-peak inflection point of load-displacement curve. From Figure 5, it was observed that the virgin asphalt mixtures ( $0 \%$ RAP) have higher fracture resistance than the RAP blended asphalt mixtures. This could be due to the reason that there is no aged RAP binder in the total mix. As the RAP proportion increased, the virgin binder content was reduced, the fracture resistance of asphalt mixtures was deteriorated.

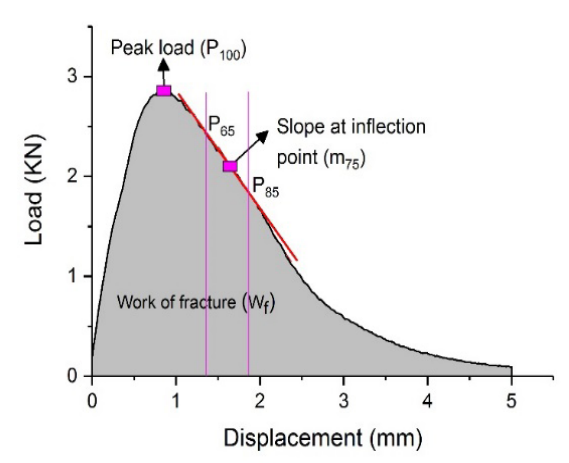

Figure 4: Load-displacement curve for $0 \%$ RAP binder (AASHTO TP 124 , 2018)

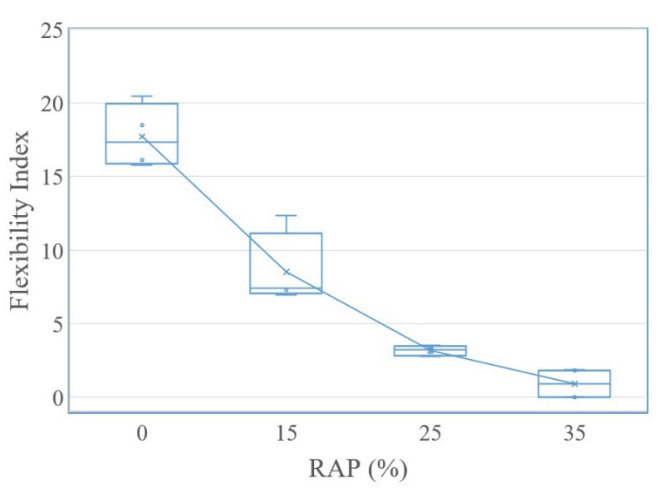

Figure 5: Flexibility Index for RAP blended asphalt mixtures 


\section{BALANCE OF PERFORMANCE}

Figure 6 shows the balancing of rut depth values (HWT dry rut depth) and fracture resistance parameter (SCB FI) of RAP blended asphalt mixtures. In Figure 6, the RAP proportion corresponding to a target rut depth of $12.5 \mathrm{~mm}$ was $18 \%$. Hence, a minimum of $18 \%$ RAP should be added to avoid failure due to rutting. In fracture test, the target FI value was considered as 8 (Mogawer et al., 2019) From Figure 6, the RAP proportion corresponding to the target FI value was marked as 16\%. Qatar has desert hot climatic conditions for many of the days in a year. Hence, it is necessary to make more rut resistant mixtures. In this study, by balancing both rutting and fracture characteristics of asphalt, it is recommended to use up to $20 \%$ of RAP without having negative impact on low temperature performance of mixtures.

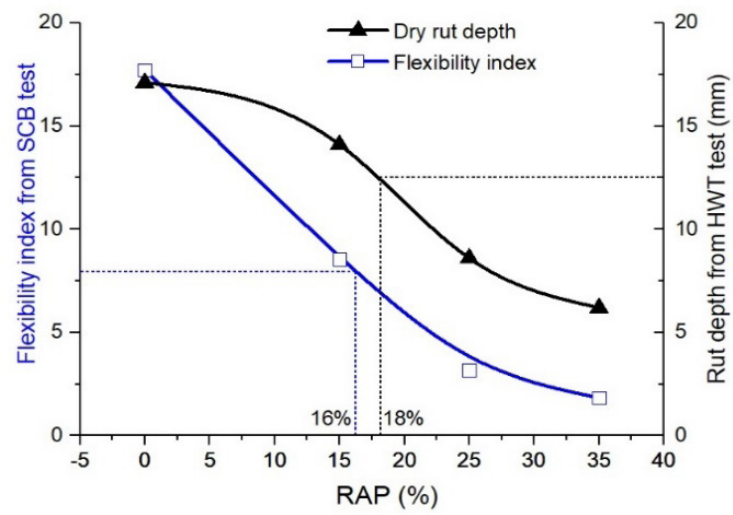

Figure 6: Balancing rutting and fracture resistance of RAP blended asphalt mixtures

\section{CONCLUSION}

The main objective of this paper was to check the influence of RAP on the performance of asphalt mixtures. The material and performance properties of asphalt mixtures were evaluated using different test protocols. First, the dynamic modulus of RAP contained asphalt mixtures were determined at different temperatures and the high and low temperature distress characteristics of RAP blended asphalt mixtures were balanced. From this investigation, the following conclusions can be drawn:

1. The asphalt mixture blended with higher proportion of RAP $(35 \%)$ has the highest dynamic modulus values and rutting resistance. However, this mixture exhibited the least fracture performance.

2. For desert hot climatic conditions of Qatar, by balancing both rutting and fracture characteristics of asphalt mixtures, it is recommended to use up to $20 \%$ of RAP without having negative impact on low temperature performance of asphalt mixtures. Given the low asphalt contents (typically less than $4 \%$ of asphalt content) in mixture designs in Qatar, and the high aging of RAP binders due to high temperatures, it is recommended to limit the RAP content to a maximum of $20 \%$ by weight of mix. The use of higher RAP contents would require changes in the asphalt mix design (binder content, gradation) and/or the use of rejuvenating agents.

\section{ACKNOWLEDGEMENT}

This investigation was supported by Consortium for Asphalt Pavement Technologies 
(CAPT), Texas A\&M at Qatar. The authors gratefully acknowledge the support of FUGRO Doha in the sample preparation and basic testing.

\section{REFERENCES}

Al-Qadi, M. Elseifi \& S. H. Carpenter (2007). Reclaimed asphalt pavement - a literature review, Tech. rep., University of Illinois at Urbana Champaign.

Francken, L. (1977). Pavement deformation law of bituminous road mixes in repeated load triaxial compression. In Fourth international conference on the structural design of asphalt pavements. University of Michigan, the USA.

Menapace, I., Cucalon, L. G., Kaseer, F., Arámbula-Mercado, E., Martin, A. E., Masad, E. \& King, G. (2018). Effect of recycling agents in recycled asphalt binders observed with microstructural and rheological tests. Construction and Building Materials, 158, 61-74.

Mogawer, W. S., Austerman, A., Roque, R., Underwood, S., Mohammad, L. \& Zou, J. (2015). Ageing and rejuvenators: evaluating their impact on high RAP mixtures fatigue cracking characteristics using advanced mechanistic models and testing methods. Road Materials and Pavement Design, 16(sup2), 1-28.

Mogawer, W. S., Austerman, A. J., Stuart, K. D., Zhou, F. \& Romero, P. (2019). Balanced mix design sensitivity to production tolerance limits and binder source. Journal of the Association of Asphalt Paving Technologist (AAPT), Vol. 88. (Under review)

Ozer, H. (2016). Development of the fracture-based flexibility index for asphalt concrete cracking potential using modified semi-circle bending test parameters. Construction and Building Materials, 115, 390-401.

Shu, X., Huang, B. \& Vukosavljevic, D. (2008). Laboratory evaluation of fatigue characteristics of recycled asphalt mixture. Construction and Building Materials, 22(7), 1323-1330.

Sreedhar, S., Coleri, E. \& Haddadi, S.S. (2018). Selection of a performance test to assess the cracking resistance of asphalt concrete materials. Construction and Building Materials, 179, 285-293.

Sreeram, A., Leng, Z., Zhang, Y., \& Padhan, R. K. (2018). Evaluation of RAP binder mobilisation 